\title{
5 years already gone for GlobalHort
}

On March 23, 2006, the Global Horticultural Initiative was launched during a special conference held in Montpellier, France, with a special focus on fostering horticulture for development (H4D) to create wealth and improve human health and well-being in the world's poorest countries through increased production, consumption, processing and marketing of fruits and vegetables and other horticultural crops. On several occasions in these editorials, we have stressed the great potential for horticulture to contribute to addressing key challenges of food security and poverty reduction through better nutrition and health, and through income generation for small farmers.

Five years later, what is the situation?

GlobalHort has carried out many actions in several areas: in promoting and advocating for Horticulture through various events such as the Awareness Raising Conference on H4D in Brussels, June 2007, and the All Africa Horticulture Congress in Nairobi, September 2009; in connecting and informing the H4D community, through its web portal www.globalhort.org; in coaching research for development projects like those on Asian Fruit Fly Management and Recipes for Success; in facilitating capacity building and knowledge management, through regional platforms in support of horticulture in Africa, through video-conferences on high-value markets in Africa and through collective actions for increasing the production and consumption of fruits and vegetables.

Despite these actions, the recognition of horticulture as an important element of development is still very low, particularly for policy-makers and donors.

Why such a situation? Since the launching of GlobalHort, there has been the triple price, food and energy crisis, culminating in 2008. Whereas before this global crisis, increasing attention was given to horticulture, one of its direct effects was a return to the hegemony of staples such as the unique solution to address food security and poverty reduction, as if there was a sleeping lobby ready to be reactivated at the earliest opportunity.

For instance, for the Bill \& Melinda Gates Foundation, "one promising solution is helping small farmers grow nutritionally enhanced staple crops like sweet potato, rice, and cassava", forgetting that there are naturally nutritious species offered by the biodiversity of our planet, but that are far from being used at their potential. And the position is not so different in international organizations for agricultural research or development.

Fortunately, there are glimmers of hope such as the joint initiative by the Global Forum for Agricultural Research (GFAR) and the Secretariat of the International Treaty on Plant Genetic Resources for Food and Agriculture (ITPGRFA) to initiate a collective action to encourage and facilitate the cultivation and marketing of a greater diversity of high-value specialty crops, both indigenous and exotic, in order to improve the incomes and health of the rural poor. Horticultural crops are part of these promising crops for development and we can only fully support Pr. Norman Looney, the GlobalHort Board Chair, when he says that "GlobalHort can contribute importantly to ensuring that high-value specialty plants and crops receive the attention deserved within the context of reducing poverty and improving food and nutrition security of smallbolders".
Dr. Jacky Ganry Scientific Director of Fruits 


\section{Déjà 5 ans pour Globalhort}

Le 23 mars 2006, l'Initiative Mondiale Horticole a été lancée lors d'une conférence spéciale tenue à Montpellier, en France. Un accent tout particulier était mis sur la promotion de l'horticulture pour le développement (H4D) en vue de créer des revenus et d'améliorer la santé humaine ainsi que le bien-être dans les pays les plus pauvres du monde au travers d'un accroissement de la production, de la consommation, de la transformation et de la commercialisation des fruits et légumes et autres espèces horticoles. À plusieurs reprises, nous avons insisté dans ces lignes éditoriales sur le très fort potentiel de l'horticulture pour contribuer à relever les principaux défis de sécurité alimentaire et réduire la pauvreté grâce à une meilleure nutrition et santé et à la création de revenus pour les petits agriculteurs.

Cinq ans plus tard, quelle est la situation ?

GlobalHort a réalisé beaucoup d'actions dans plusieurs domaines: dans la promotion et la défense de l'horticulture au travers de diverses manifestations telles que la Conférence de Sensibilisation sur $H 4 D$ à Bruxelles en juin 2007 et le Congrès sur l'Horticulture en Afrique, à Nairobi, en Septembre 2009; en reliant et en informant la communauté de l'Horticulture pour le Développement, grâce à son portail web www.globalhort.org; dans le "coaching" de la recherche pour des projets de développement tels que ceux sur le contrôle des Mouches des Fruits en Asie et Les recettes pour réussir; en facilitant le renforcement des capacités et la gestion des connaissances, par le biais de plates-formes régionales en appui à l'horticulture en Afrique, de vidéoconférences sur les marchés rémunérateurs en Afrique et à travers des actions collectives pour accroître la production et la consommation de fruits et légumes.

En dépit de ces actions, la reconnaissance de l'horticulture comme un élément important du développement est encore très faible, en particulier pour les décideurs et les bailleurs de fonds.
Pourquoi une telle situation? Depuis le lancement du GlobalHort, il y a eu la triple crise financière, alimentaire et énergétique qui a culminé en 2008. Alors que, avant cette crise mondiale, une attention croissante était accordée à l'horticulture, l'un de ses effets directs a été un retour à l'hégémonie des aliments de base comme la solution unique pour aborder la sécurité alimentaire et la réduction de la pauvreté, comme s'il y avait un lobbying dormant prêt à être réactivé à la première occasion.

Par exemple, pour la Fondation Bill \& Melinda Gates, "une solution prometteuse est d'aider les petits agriculteurs à cultiver des cultures de base améliorées sur le plan nutritionnel comme la patate douce, le riz et le manioc", oubliant qu'il existe des espèces naturellement riches au plan nutritionnel, offertes par la biodiversité de notre planète, mais qui sont loin d'être utilisées à leur plein potentiel. Et la position n'est pas si différente dans les organisations internationales de recherche agricole ou de développement.

Heureusement, il y a des lueurs d'espoir comme l'initiative conjointe du Forum mondial pour la recherche agricole (GFAR) et le Secrétariat du Traité international sur les ressources phytogénétiques pour l'alimentation et l'agriculture (ITPGRFA) pour lancer une action collective visant à encourager et faciliter la culture et la commercialisation d'une plus grande diversité d'espèces à valeur ajoutée, à la fois indigènes et exotiques, afin d'améliorer les revenus et la santé des populations rurales pauvres. Les cultures horticoles font partie de ces cultures prometteuses pour le développement et nous ne pouvons que soutenir pleinement le Pr. Norman Looney, président du conseil de GlobalHort, quand il dit que "GlobalHort peut largement contribuer à ce que les plantes et cultures spécialisées à forte valeur ajoutée reçoivent l'attention qu'elles méritent dans une perspective de réduction de la pauvreté et d'amélioration de la sécurité alimentaire et de la nutrition des petits exploitants».

Dr. Jacky Ganry Directeur scientifique de Fruits 